\title{
Effect of size and chemical composition of graphene oxide nanoparticles on optical absorption cross-section
}

\author{
Mohadeseh Hashemi \\ Bharadwaj Muralidharan \\ Meisam Omidi \\ Javad Mohammadi \\ Yahya Sefidbakht \\ Eun Song Kima \\ Hugh D.C. Smyth \\ Mohammad Shalbaf \\ Thomas E. Milner
}




\title{
Effect of size and chemical composition of graphene oxide nanoparticles on optical absorption cross-section
}

\author{
Mohadeseh Hashemi, ${ }^{a, b, c}$ Bharadwaj Muralidharan, ${ }^{a, d}$ Meisam Omidi, ${ }^{e, *}$ Javad Mohammadi, ${ }^{a, b, *}$ \\ Yahya Sefidbakht, ${ }^{e}$ Eun Song Kima, ${ }^{c}$ Hugh D.C. Smyth, ${ }^{c}$ Mohammad Shalbaf, ${ }^{e}$ and Thomas E. Milner ${ }^{a, *}$ \\ aUniversity of Texas at Austin, Department of Biomedical Engineering, Austin, Texas, United States \\ bUniversity of Tehran, Faculty of new Science and Engineering, Tehran, Iran \\ 'University of Texas at Austin, Division of Pharmaceutics, College of Pharmacy, Austin, Texas, United States \\ dUniversity of Texas at Austin, Department of Electrical and Computer Engineering, Austin, Texas, United States \\ eShahid Beheshti University, G.C., Protein Research Center, Tehran, Iran
}

\begin{abstract}
Photothermal therapy with various nanoparticles, as photothermal transducers, is a widely researched technique. A continuous wave (CW) laser is employed during this procedure. The therapeutic setup is slightly modified to measure the optical absorption cross-section of the graphene oxide (GO), by mitigating the effects of heat diffusion and light scattering. With an 808-nm CW laser setup modulated by a waveform modulation setup, the effect of nanoparticle size and composition of GO in water on optical absorption cross section is characterized. () 2018 Society of Photo-Optical Instrumentation Engineers (SPIE) [DOI: 10.1117/1.JBO.23.8.085007]
\end{abstract}

Keywords: graphene oxide; cross-section absorption; absorption coefficient; laser.

Paper 180071R received Feb. 8, 2018; accepted for publication Aug. 3, 2018; published online Aug. 28, 2018.

\section{Introduction}

Photothermal therapy is a medical procedure based on light-toheat conversion for selective destruction of targeted cells. Considering the entire spectral range from ultraviolet (UV) to infrared (IR), the near-IR (NIR) region provides some advantages for photothermal therapy since absorption by water is small and light has a relatively deep penetration depth in tissue $^{1-10}$ A critical component of photothermal therapy is a photothermal transducer that can absorb and convert NIR light into heat with high efficiency..$^{2-8,10-15}$ In recent years, graphene oxide $(\mathrm{GO})$ has received interest as a potential photothermal transducer due to excellent biocompatibility, high loading efficiency, and the capability of conjugating a large variety of functional groups on the surface to target various cell types. ${ }^{1,10,11,16-24}$

An important property of a photothermal transducer is absorption cross section, which determines the photothermal potential of an agent to absorb incoming light. ${ }^{1,25,26}$ Among the graphene family, the absorption cross section of graphene at $660 \mathrm{~nm}$ has been reported by Yi et al. and Khan et al. ${ }^{18,27}$ by utilizing the slope of the absorbance per unit cell length against the concentration of the suspension in the cell. However, there was a difference in the reported value for graphene. Yi et al. ${ }^{20}$ reported absorption cross section of graphene at $660 \mathrm{~nm}$ to be $36.00 \mathrm{ml} /(\mathrm{mg} . \mathrm{cm})$, at a lower concentration (20 to $40 \mu \mathrm{g} / \mathrm{ml}$ ) based on the Lambert-Beer law. While Khan et al. showed that at higher graphene concentrations of 0.1 to $1 \mathrm{mg} / \mathrm{ml}$, the absorption cross section can vary from 24.60 to $36.20 \mathrm{ml} /(\mathrm{mg} . \mathrm{cm})$ and is relatively insensitive to processing procedure. ${ }^{18,27}$ However, the reason behind these variation in

\footnotetext{
*Address all correspondence to: Meisam Omidi, E-mail: m_omidi@sbu.ac.ir; Javad Mohammadi, E-mail: drjmohamadi@yahoo.com; Thomas E. Milner, E-mail: terra.laser@gmail.com
}

reported results have not been accounted. It should be worth noted that the values reported by Khan et al. and Yi et al. for graphene was molar absorptivity or molar attenuation coefficient $\left(\frac{\mathrm{ml}}{\mathrm{mg} . \mathrm{cm}}\right)$ while absorption cross section is absorptivity $\times$ $\frac{10^{3} \times \log _{10}(e)}{\text { Avogadro number }}\left(\mathrm{cm}^{2}\right)$, where absorptivity is expressed in decadic form.

Ideally, with no scattering involved, the absorption cross section should remain the same irrespective of the concentration or the size of the nanoparticle in the suspension and vary with carbon to oxygen content of the GO nanoparticles. However, in the suspension, scattering cannot be completely eliminated. Scattering will affect absorption profile (or, the noise floor) of the detected signal with IR camera. As shown in Eqs. (1) and (3), increase in scattering increases the energy lost to the surroundings, typically due to scattering and heat diffusion (represented as ${ }^{\circ} Q_{\text {lost }}$ ), thereby reducing ${ }^{\circ} Q_{\text {in }}$ and ${ }^{\prime} Q_{\mathrm{w}}$. This, in turn, would affect the accuracy of computations of absorption coefficient and thereby the absorption cross-section values.

The accuracy in calculation of the absorbance per unit length (or absorption coefficient) by Lambert-Beer law depends on several factors, such as the particle size and concentration and the system noise. As the concentration of the suspension in consideration is increased, the chances of scattering increase. This brings in more uncertainty in accuracy of computing absorbance as the chances of reabsorption of the scattered light increases with concentration. Moreover, the effect of noises in the measurement system on the computation is an unknown factor and might affect the computed slope. In order to reduce these uncertainties, development of in situ measurement technique was necessary.

The in situ system is designed to evaluate the absorption coefficient of nanoparticle suspension under reduced

$1083-3668 / 2018 / \$ 25.00$ (c) 2018 SPIE 
uncertainties. First, the concentration of the suspension for a given particle size is fixed to reduce the loss due to scattering. The highest concentration of the suspension with a similar scattering profile as that of an optical standard and/or a standardized absorber (with a known/computed scattering to absorption percentage) is considered for estimation of absorption coefficient. Second, the heat lost to the surroundings by diffusion process is minimized by utilizing a pulsed laser over to a continuous laser, with a pulse duration far less than the thermal relaxation time of the nanoparticle suspension. Pulses were generated by modulating the current to the laser diode [continuous wave (CW) laser], thereby controlling the power and pulse duration of the laser diode. Moreover, utilizing the filters and Fourier domain computation, the effects of noises such as laser intensity fluctuations and external environment were minimized.

\section{Theory}

In general, the energy balance equation for laser energy absorption rate $\left(\dot{Q}_{\text {in }}\right)$ of single nanoparticles in suspension can be written as $^{28}$

$\dot{Q}_{\text {in }}=V_{g} \rho_{g} C_{g} \frac{d T_{g}}{d t}=V_{g} \mu_{\alpha} \phi(t)-\dot{Q}_{\text {lost }}$.

In Eq. (1), $V_{g}$ is nanoparticles volume, $\rho_{g}$ and $C_{g}$ are nanoparticles density and specific heat capacity, and $T_{g}$ and $t$ are nanoparticles temperature and time, respectively. On the right side of Eq. (1), $\mu_{\alpha}$ is the absorption coefficient of the nanoparticles, $\phi(t)$ is the laser beam of fluence, and $\dot{Q}_{\text {lost }}$ is the heat loss rate. The term for heat loss rate $\dot{Q}_{\text {lost }}$ includes rate of energy lost from light backscattered from the surface and transmitted through the sample and thermal heat loss rate to surroundings of the nanoparticles. For laser pulse duration shorter than thermal diffusion rate, the effect of heat loss rate is neglected. ${ }^{29}$ When the laser pulse is turned off, the absorbed heat energy will begin to equalize with the surroundings, and the energy conservation equation can be expressed as

$\dot{Q}_{w}=\dot{Q}_{g}=V_{g} \rho_{g} C_{g} \frac{d T_{g}^{\prime}}{d t}=m_{w} C_{w} \frac{d T_{w}}{d t}$,

$\dot{Q}_{w}$ and $\dot{Q}_{g}$ denote the energy variation rate of water and nanoparticles, and $m$ and $C$ are the mass and the calorific value, the subscripts "w" and "g" stand for water and nanoparticles, respectively. By the following assumption that, all the absorbed laser energy in irradiation time would be transferred to the surrounding medium, the new form of the energy conservation equation can be expressed as

$\dot{Q}_{\mathrm{w}}=\dot{Q}_{\mathrm{in}} \rightarrow V_{\mathrm{g}} \mu_{\alpha} \phi(t)=m_{\mathrm{w}} C_{\mathrm{w}} \frac{d T_{w}}{d t}$.

Equation (3) in term of surrounding medium temperature variation rate can be rewritten as

$$
\frac{d T_{\mathrm{w}}}{d t}=\frac{w_{\mathrm{g}} \mu_{\alpha} \phi(t)}{\rho_{\mathrm{g}} C_{\mathrm{w}}},
$$

where $w_{\mathrm{g}}=m_{\mathrm{g}} / m_{\mathrm{w}}$ is a weight ratio. The absorption cross section of the nanoparticles can be obtained from the absorption coefficient $^{30}$ as
$\sigma=\frac{\mu_{\alpha}}{N}=\frac{\mu_{\alpha}}{\rho_{b}} \frac{m_{a}}{N_{a}}$

where $N$ is the atomic or molecular number density (or, number of particles in unit volume), $\rho_{b}$ is the bulk density, $m_{a}$ is the molecular mass, and $N_{a}$ is the Avogadro's number. The molecular dynamics (MD) simulation method ${ }^{31}$ was applied for calculation of atomic molar mass and nanoparticles density. ${ }^{32-35}$

A time-domain approach to calculate the absorption cross section may be used, but the computation accuracy might be influenced by the fluctuations that can arise from laser intensity and external environmental variations. The lateral diffusion is dependent on the thermal relaxation time. The thermal relaxation time is given as

$\tau=\frac{d^{2}}{16 x}$

where $x$ is the thermal diffusivity of the dispersing media $\left(\mathrm{mm}^{2} / \mathrm{s}\right)$ and $d$ is the diameter of the laser beam spot size on the sample $(\mathrm{mm})$. The laser modulation is set to a frequency whose time period is less than the relaxation time of the suspension to ensure thermal relaxation does not occur during the experiment. While the minimum limit on the frequency of modulation is set by the relaxation time of the suspension, the maximum is dependent on the maximum frame rate of the IR camera for the acquisition.

The light scattering by nanoparticles is an unwanted effect for computing the absorption cross section. Although the scattering cannot be avoided, it can be reduced. The scattering model for the nanoparticles is dependent on the size of the particles with respect to the irradiating wavelength. ${ }^{36}$ If the nanoparticle of average radius $a$ is excited by the laser wavelength $\lambda$, the size parameter, $a_{r}$, and the effective radius, $a$ are given as

$a_{r}=\frac{2 \pi a}{\lambda}$,

$a=\left(\frac{3 V}{4 \pi}\right)^{1 / 3}$,

where $V$ is the volume of a particle with an arbitrary shape. ${ }^{30}$ The GO sheets have the planar geometry and its effective radius is given by Eq. (7), which can be rewritten as

$a_{r}=\frac{2 \pi(3 * l * s * t / 4 \pi)^{1 / 3}}{\lambda}$,

where $l$ and $s$ are sheet lateral dimensions and $t$ is the thickness of GO sheet.

Scattering of light by a spherical nanoparticle of any size can be modeled with Mie scattering theory. However, if the size parameter $a_{r} \ll 1$ (as in ultrasmall nanoparticle case), the model reduces to Rayleigh scattering model. For original GO particle (nearly 1 to $5 \mu \mathrm{m}$ ), the particle is big in comparison to $\lambda$. Hence, the diffraction effects of light rays need to be accounted, giving rise to variabilities in absorption cross-section computation by the experiment. Irrespective of the model, the intensity of the backscattered light from the sample increases with the size and the concentration of the particles. The backscattered light might reduce the energy available for absorption, as some of the backscattered light will scatter back from the 
surface of the sample. Moreover, this backscattered light could alter the observed temperature, as measured by the IR camera, giving rise to possible erroneous results for absorption cross section. Thus, it is preferable to reduce the backscattering by reducing the nanoparticles size (preferably, an order lower than $\lambda$, i.e., $\left.a_{r} \leq 0.1 \Rightarrow a \leq \lambda / 20 \pi\right)$. At 808 -nm wavelength, the effective radius $a$ is $128.6 \mathrm{~nm}$ for $a_{r}=1$. This implies that the effect of diffraction needs to be considered if the particle radius is $>128.6 \mathrm{~nm}$. Thus, based on Eq. (9), the product of particle dimension should satisfy the condition to consider Rayleigh scattering model for 808 -nm wavelength excitation: $(3 * l * s * t / 4 \pi)^{1 / 3} \leq 12.9 \mathrm{~nm}$.

The scattering cross section $\left(\sigma_{\mathrm{sca}}\right)$ of a spherical particle, based on the Rayleigh scattering model, is directly proportional to $a^{6}$ with backscattering as similar to the forward scattering profile. ${ }^{37}$ Reducing scattering reduces the chances of multiple scattering and reabsorption of scattered light by nanoparticles. Assuming the heat loss, such as from lateral diffusion, is negligible, the reduction in scattering, by utilizing smaller particles size (typically, 10 to $100 \mathrm{~nm}$ in diameter), leads to an increase in the intensity of the light available for absorption. Moreover, the experimental results of UV-vis spectroscopy show that reducing the GO nanoparticle size increases the absorbance of the sample at equivolume and concentration, corroborating with observations by Robinson et al. ${ }^{38}$ This increase in absorbance increases the computed absorption coefficient and absorption cross section. Assuming no other loss, the efficiency factor of absorption $\left[\eta_{\text {abs }}=\sigma /\left(4 \pi a^{2}\right)\right]$ increases as the efficiency factor of scattering $\left[\eta_{\text {sca }}=\sigma_{\text {sca }} /\left(4 \pi a^{2}\right)\right]$ reduces, as given as

$\eta_{\mathrm{ext}}=\eta_{\mathrm{abs}}+\eta_{\mathrm{sca}}$,

where $\eta_{\text {ext }}$ is the efficiency factor of the incident laser beam.

Moreover, the scattering coefficient $\left(\mu_{\text {sca }}\right)$ is proportional to the concentration of the nanoparticles. Thus, reducing the concentration of the nanoparticles reduces scattering and the chances of multiple scattering and reabsorption, thereby increasing the accuracy in calculating absorption cross section by this method described in Sec. 3. However, the concentration cannot be reduced so low that the thermal signature of the nanoparticle is undiscernible by the IR camera from its surroundings. Thus, there is a trade-off that is necessary in deciding the concentration required for conducting the experiment for calculating the absorption cross section-thermal signal and scattering coefficient.

The time-domain analysis to compute the absorption cross section, using the above concepts and equations, can be implemented. However, the time-domain analysis is prone to the fluctuations that can arise from the external environment and/ or the laser intensity variation. Hence, it is preferable to use a Fourierdomain analysis with a modulating signal. Utilizing the analytic signals for the temperature and laser fluence, $T=T_{0} e^{i 2 \pi f t}$, and $\varphi=\varphi_{0} e^{i 2 \pi f t}$, respectively, and applying it to the magnitude of the Fourier-domain equivalent of Eq. (3), the absorption coefficient of the sample is given as

$\mu_{\alpha}=\frac{2 \pi f \rho_{\mathrm{g}} C_{\mathrm{w}} T_{0}}{w_{\mathrm{g}} \phi_{0}}$.

Since the absorption coefficient and particle concentration of the sample are not a time-dependent component, Eq. (5) can be used to compute the absorption cross section of the nanoparticles.

\section{Methods}

The absorption cross section of the nanoparticles is measured by modulating the power of the CW laser of the therapeutic setup, after minimizing the scattering of the nanoparticles. The modulation frequency is determined after calculating the relaxation time. The therapeutic setup and the modifications for computing absorption cross section are described below. Also, molecular dynamic simulation is provided to calculate the molar mass and density of the particles.

\subsection{GO Preparation}

In this study, the two different types of GO with oxygen-to-carbon ratio of 0.12 and 0.21 were provided by graphene supermarket. Both types of stock GO were sonicated, using a probe sonicator (Sonicator 3000, Misonix Inc., New York) at a total power output of $100 \mathrm{~W}$ with different operation time durations ( 1 and $2 \mathrm{~h}$ ), to produce different sized GO sheets samples. To monitor size reduction, atomic force microscopy (AFM) (SPM, Veeco Instruments Inc.) operating at tapping mode was utilized. The optical density of an aqueous dispersion of GO $(40 \mu \mathrm{g} / \mathrm{ml})$ in each time interval of the experimental procedure has been obtained using UV-vis spectroscopy (Infinite M200, Tecan Systems Inc.). The Raman spectrum of GO samples with 514-nm excitation was recorded using an Alpha 300 spectrometer (WITec GmbH, Germany).

\subsection{Therapeutic Setup}

The therapeutic setup consists of a CW laser diode, emitting at $808 \mathrm{~nm}$, which is coupled into multimode optical fiber (BFL481000, Thorlabs Inc.). Laser diode current and temperature controllers (LDC 240C and TED 200C respectively, Thorlabs, Inc.) aide in maintaining constant emission wavelength and output optical power of the laser diode. The end face of multimode optical fiber is imaged to a spot size of $6 \mathrm{~mm}$ diameter on the surface of the GO suspension, with an aspheric condenser lens $(f=20.35 \mathrm{~mm})$ (ACL2520U-B, Thorlabs Inc.). The temperature of the GO suspension was recorded using a temperature calibrated InSb IR camera (FLIR systems, Inc., SC4000 MWIR).

\subsection{Measuring Absorption Cross Section and Scattering Profile}

The setup to measure the absorption cross section includes providing a modulation signal to the laser diode current controller and synchronizing IR camera. The modulation signal sent to the laser diode current controller controls the power of the laser, thereby making the $\mathrm{CW}$ laser-a long duration pulsed laser. The modulation frequency is dependent on the thermal relaxation time of the nanoparticle. Acquisition control signals for IR camera synchronize the frame captured with laser operation. The modulation signals and frame acquisition controls were provided by two function generators (33250A, Agilent Inc. and model 645, Berkeley Nucleonics Corp.) and a digital delay generator (DG535, Stanford Research Systems, Inc.). The same setup is also used for characterizing the scattering profile of the nanoparticles. However, for characterizing the scattering profile, the modulation signal to the current controller modifies the CW laser to produce a short pulse and an image is acquired at the end of the pulse with the help of a synchronized frame acquisition signal to the IR camera. For this study, electronic 
control was preferred as it provides accurate control on modulation frequency. The schematic of the control has been provided in Appendix.

\subsection{Characterization of Black Absorber}

A 250-nm thick black absorber is used for comparison with GO nanoparticles dispersion solution. The scattering of this black absorber was characterized by comparing it with an optical diffusive standard (WS1 reflective standard, Ocean optics Inc.). The above-mentioned 808-nm CW laser setup was modulated by a square wave, with modulation signal parameters being the same as in the calculation of the absorption cross section. The beam from the multimode fiber, collimated by an aspheric condenser lens ( $f=20.35 \mathrm{~mm}$ ) (ACL2520U-B, Thorlabs Inc.), was positioned at the end of a port of an integration sphere (general purpose integrating sphere, Labsphere Inc.). The test material at the second port of this integration sphere was irradiated by the collimated beam from the first port. A power meter (StarLab suite, Ophir Optronics Solutions Ltd.) was placed at the third port of the integrating sphere to measure the power at the port.

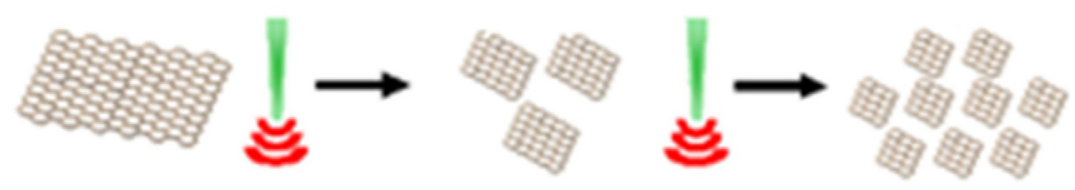

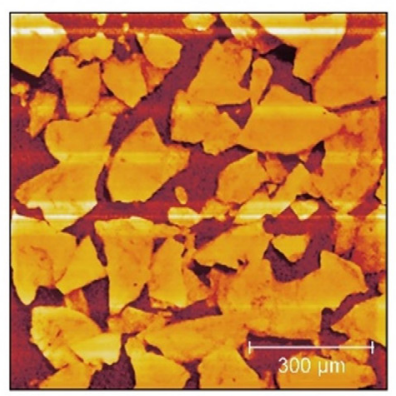

(a)

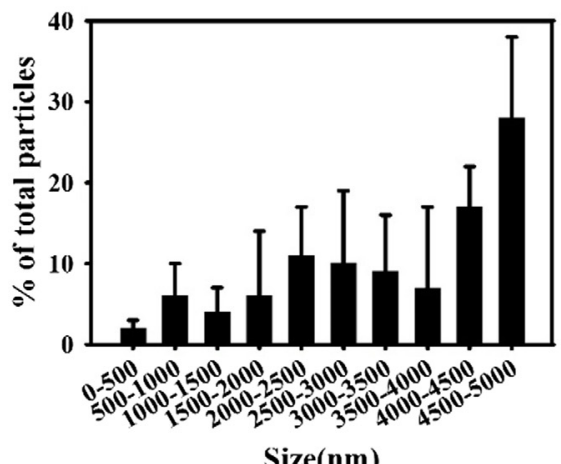

(d)

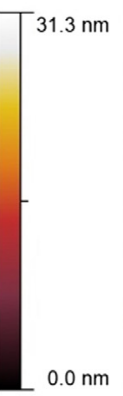

$$
{ }^{40}{ }^{40}
$$

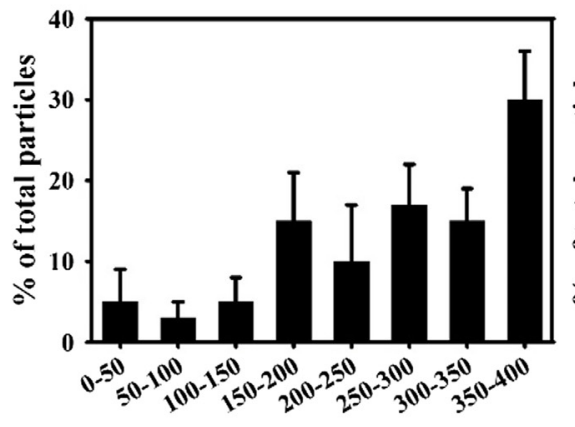

$\operatorname{Size}(\mathrm{nm})$

(e)

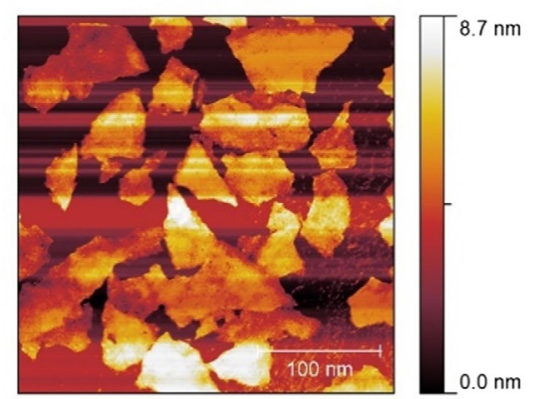

(c)

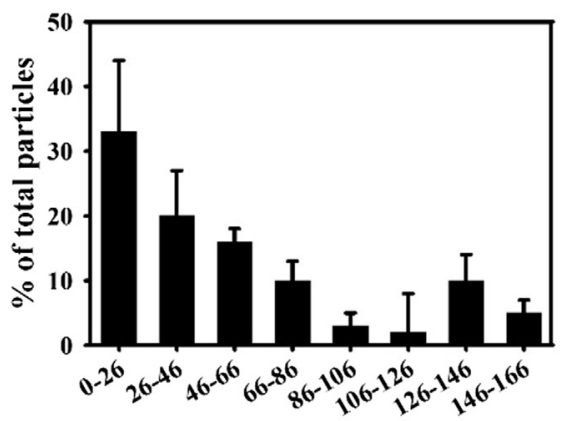

Size(nm)

(f)

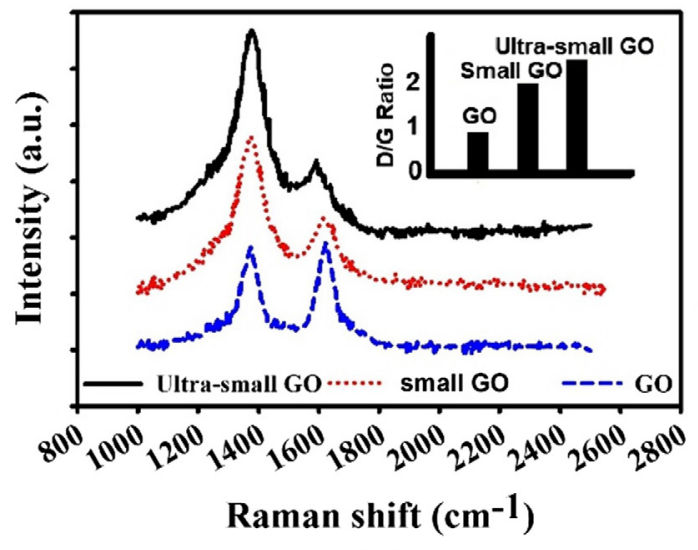

(g)

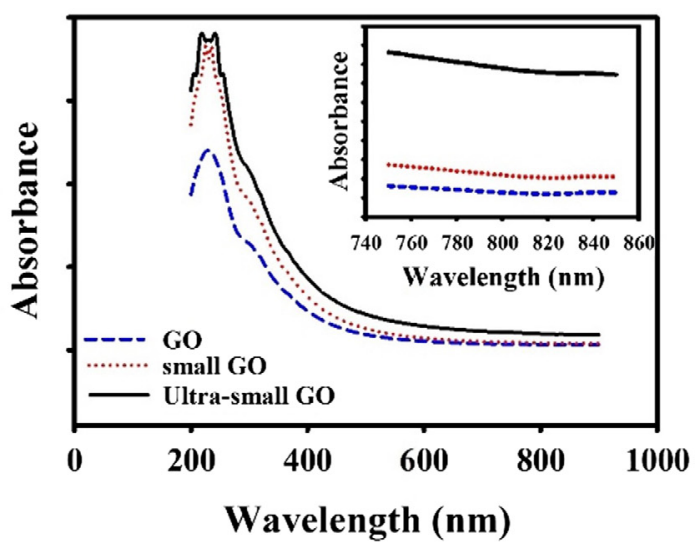

(h)

Fig. 1 AFM image of (a) GO, (b) small GO, and (c) ultrasmall GO, PDI image of (d) GO, (e) small GO, and (f) ultrasmall GO (scale bar is $100 \mathrm{~nm}$ ), (g) Raman spectra, and (h) UV-vis spectra of the samples. 


\subsection{Molecular Dynamics Simulation}

The GO coordinates were built using visual molecular dynamics (University of Illinois at Urbana-Champaign) and open source molecular editor software, Avogadro 1.90.0 (Avogadro development team). The structure was then minimized with the gromos54A7 force field using automated force field topology builder. ${ }^{32,33}$ MD simulations were carried out using GROMACS 5.1.4 simulation package to study the dynamics of GO in aqueous phase. ${ }^{34,35}$ All carboxylate groups were deprotonated, and for water molecules, the SPC model was used in a cubic box with at least $3-\mathrm{nm}$ distance from the both 8 to $12 \mathrm{~nm}$ and 120 to $160 \mathrm{~nm}$ rectangular GO sheet (positioned at the center). The simulation box was filed with $\mathrm{NaCl} 0.01 \mathrm{M}$ to satisfy ionic strength and neutralization condition. ${ }^{31}$ Initial energy minimization, followed by equilibration at NVT (constant number of particles, volume, and temperature) and NPT (constant number of particles, pressure, and temperature) were performed on the system and then MD was performed at $300 \mathrm{~K}$ over $5 \mathrm{~ns}$. The trajectory was then analyzed for deviation (RMS value) to estimate the atomic molar mass and density using GROMACS tools. ${ }^{33}$

\section{Results and Discussion}

\subsection{GO Characterization}

The size of nanosheets was decreased, by 1 and $2 \mathrm{~h}$ sonication, to produce small GO and ultrasmall GO, respectively. The morphology and polydispersity index (PDI) of GO sheets were characterized by AFM (Fig. 1). Images indicate that the size reduction of GO sheets is consistent with increased sonication time. Prior to sonication, the lateral size of GO sheets ranged from 0.5 to $5 \mu \mathrm{m}$ [57\% above $3 \mu \mathrm{m}$, Figs. 1(a) and 1(d)] while $1 \mathrm{~h}$ sonicating period reduced the size of sheets to a range of 20 to $400 \mathrm{~nm}$ with average size of $166 \mathrm{~nm}$ [Figs. 1(b) and 1(c)]. By increasing the sonication time to $2 \mathrm{~h}$, the size spread reduced further ranging from 6 to $166 \mathrm{~nm}$ with average size of $12 \mathrm{~nm}$ [Figs. 1(c) and 1(f)]. Furthermore, defect formation during sonication process was investigated by Raman spectroscopy [Fig. 1(d)]. Before sonication, GO spectra showed two distinctive peaks at $\sim 1370$ and $\sim 1620 \mathrm{~cm}^{-1}$, which correspond to D and $G$ bands, respectively. A 2-h sonication shifted the $G$ band toward higher values due to the oxygenation of the functional group. In addition, the ID/IG ratio increased from 0.81 for presonication to 1.89 and 2.4 after 1 and $2 \mathrm{~h}$ of sonication, respectively, suggesting GO defects increase with longer probe sonication. ${ }^{1,17,39}$ Then, samples have been examined by UVvis spectroscopy. As shown in Fig. 1(e), ultrasmall GO (with an average size of $\sim 12 \mathrm{~nm}$ ) has a higher optical absorbance as compared to small GO (with an average size of $\sim 166 \mathrm{~nm}$ ) and GO, especially around $800 \mathrm{~nm}$ wavelength (inset picture). The increase of dispensability properties of ultrasmall GO may increase light absorption during spectroscopy.

\subsection{Minimizing Heat Loss Through Lateral Diffusivity}

A sample holder (in this case, a standard 96-well plate) with enough breadth and depth was utilized, ensuring the scattered light through the walls of the sample holder is negligible. Time necessary for heat loss through lateral diffusion was computed according to Eq. (6). An appropriate laser pulse far shorter than the time for lateral heat diffusion was chosen, thereby mitigating the effects of lateral heat diffusion.
Thus, for a thermal diffusivity of water, the lateral thermal relaxation time was computed to be $1.78 \mathrm{~s}$ (sample spot size of $2 \mathrm{~mm}$ diameter is chosen to ensure that reflection of the light is minimized from the boundary of sample with the sample holder). So, a laser pulse repetition rate of $2 \mathrm{~Hz}$ was chosen with a $50 \%$ duty cycle so that the pulse duration of $250 \mathrm{~ms}$ is well below the lateral thermal relaxation time.

\subsection{Minimizing Heat Loss Through the Scattering Effect}

To mitigate the effects of heat loss owing to light scattering, a time series of spatial temperature variation was recorded using the IR camera at different concentrations of both small and ultrasmall GO $(0.25,0.5$, and $1 \mathrm{mg} / \mathrm{ml})$ placed in the sample holder and compared to the corresponding profile of a standardized black (at $808 \mathrm{~nm}$ ) absorber. First images of each sample after emission of a 25 -ms laser pulse were used to obtain the variation of the surface temperature along the diameter of the surface profile. Since the lateral heat diffusion has azimuthal symmetry, the profile was fitted to a Gaussian curve. The sample with a similar lateral profile to that of a characterized black absorber against an optical standard (backscattered intensity of black absorber is $9.8 \%$ of the incident intensity) was chosen to compute cross-section absorption. To find the optimized concentration of both small and ultrasmall GO to reduce scattering effect, the temperature profile of various small and ultrasmall GO concentration was compared to a reference black absorber [Figs. 2(b)-2(d)]. The optimized concentration of both small and ultrasmall GO that meet the aforementioned condition was $0.25 \mathrm{mg} / \mathrm{ml}$ (see $C$ parameter in Table 1).

As mentioned in Sec. 1, the total energy lost due to scattering from the GO suspension is dependent on two factors of the suspension: size of the particles and the concentration of the suspension. When computing the absorption cross section of nanoparticles by using Lambert-Beer law, absorbance of equal volume of different concentration suspension (samesized particles) is used. However, the energy lost due to scattering of different concentration suspensions is different, and this variation needs not be linear. This means the accuracy of this slope (absorption coefficient) of the absorbance per unit length against concentration curve is variable due to the concentration range under experimentation. Moreover, this slope value can drastically change with the particle size in consideration.

However, by evaluating the heat loss due to scattering and fixing the loss against a standardized scattering material, the concentration of the suspension, for the sample with a range of particle size, to be used for the computation of absorption coefficient is fixed. This characterization fixes the energy available for absorption of heat by the GO suspension. This implies that the concentration of the GO suspension used for computation of absorption coefficient should be smaller as the particle size increases for a fixed heat loss due to scattering.

In ideal case, irrespective of the particle size and concentration, the absorption coefficient should be same across different samples for a fixed input fluence. Hence, as long as the radius of particles are smaller than $\lambda / 2 \pi$, the results should theoretically remain the same. However, as shown in Table 1, the variations in spatial temperature spread are larger for larger-sized particles. This variation can be attributed to the variations in particle size as suggested by PDI from AFM analysis of the samples. Typically, the larger (e.g., small-sized particles) sized particle batch are produced by sonication of the original material for 
(a)
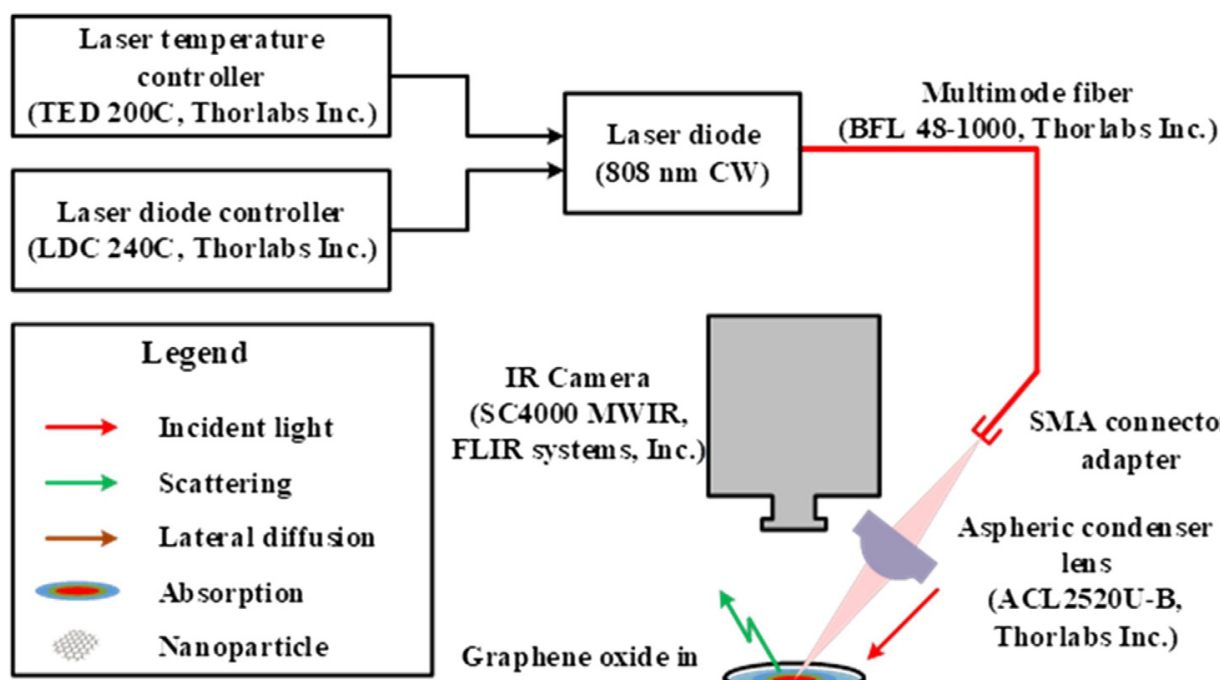
(808 nm CW) Graphene oxide i
aqueous solution

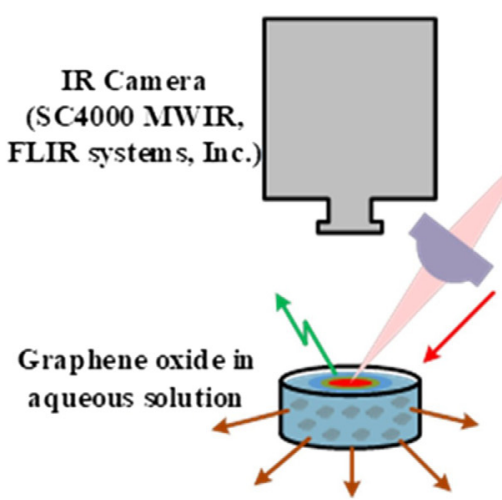

SMA connector adapter

Aspheric conden ser

lens

(ACL2520U-B,

Thorlabs Inc.)
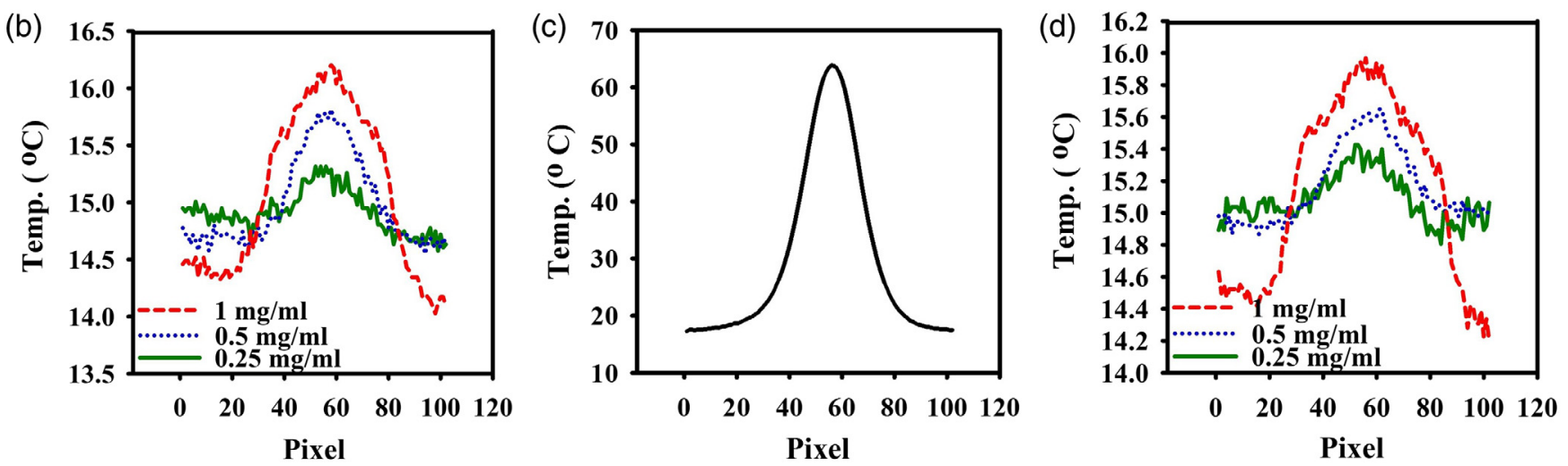

Fig. 2 (a) Schematic of setup to measure absorption cross section. Scattering profile of (b) Ultra-small GO at different concentration, (c) black paper, and (d) small GO at different concentration. The resolution of the CCD array of the IR camera is $38.8 \mu \mathrm{m} /$ pixel with a noise equivalent temperature difference of $18 \mathrm{mK}$.

Table 1 Scattering evaluation of small and ultrasmall GO at various concentration $T(x)=a e^{\left[-(x-b / c)^{2}\right]}+d$.

\begin{tabular}{|c|c|c|c|c|c|c|c|}
\hline \multirow[b]{2}{*}{ Parameters } & \multicolumn{3}{|c|}{ Small GO } & \multicolumn{3}{|c|}{ Ultrasmall GO } & \multirow[b]{2}{*}{ Black pape } \\
\hline & $0.25 \mathrm{mg} / \mathrm{ml}$ & $0.5 \mathrm{mg} / \mathrm{ml}$ & $1 \mathrm{mg} / \mathrm{ml}$ & $0.25 \mathrm{mg} / \mathrm{ml}$ & $0.5 \mathrm{mg} / \mathrm{ml}$ & $1 \mathrm{mg} / \mathrm{ml}$ & \\
\hline$a$ & $0.3765 \pm 0.052$ & $0.674 \pm 0.02$ & $1.75 \pm 0.2$ & $0.507 \pm 0.025$ & $1.155 \pm 0.01$ & $2.108 \pm 0.11$ & 45.32 \\
\hline$b$ & $55.87 \pm 1.51$ & $57.34 \pm 1.97$ & $55.37 \pm 0.79$ & $52.667 \pm 0.79$ & $54.695 \pm 0.51$ & $53.525 \pm 0.61$ & 56.16 \\
\hline$c$ & $13.81 \pm 2.37$ & $18.29 \pm 1.54$ & $31.74 \pm 2.98$ & $13.84 \pm 1.02$ & $17.522 \pm 0.87$ & $27.812 \pm 1.722$ & 14.92 \\
\hline$d$ & $15.01 \pm 0.02$ & $14.95 \pm 0.02$ & $14.2 \pm 0.14$ & $14.595 \pm 0.02$ & $14.53 \pm 0.02$ & $14.132 \pm 0.09$ & 18.22 \\
\hline
\end{tabular}

lesser time, which end up having larger variations in particle size distribution within the batch.

\subsection{Absorption Cross Section}

As shown in Eq. (5), the atomic molar mass and GO density are needed for calculating the absorption coefficient and absorption cross section. The $m_{a}$ and $\rho_{g}$, obtained from MD simulation, are dependent on GO sheet size and oxygen to carbon ratio. The average GO sheet size for small and ultrasmall GO was obtained from Fig. 1, and results were presented in Table 3. The magnitude of the absorption coefficient was calculated on a laser pulse basis. To measure the temperature per pulse, $T_{0}, 400 \mu \mathrm{l}$ of $0.25 \mathrm{mg} / \mathrm{ml}$ of small and ultrasmall GO with oxygen-to-carbon 

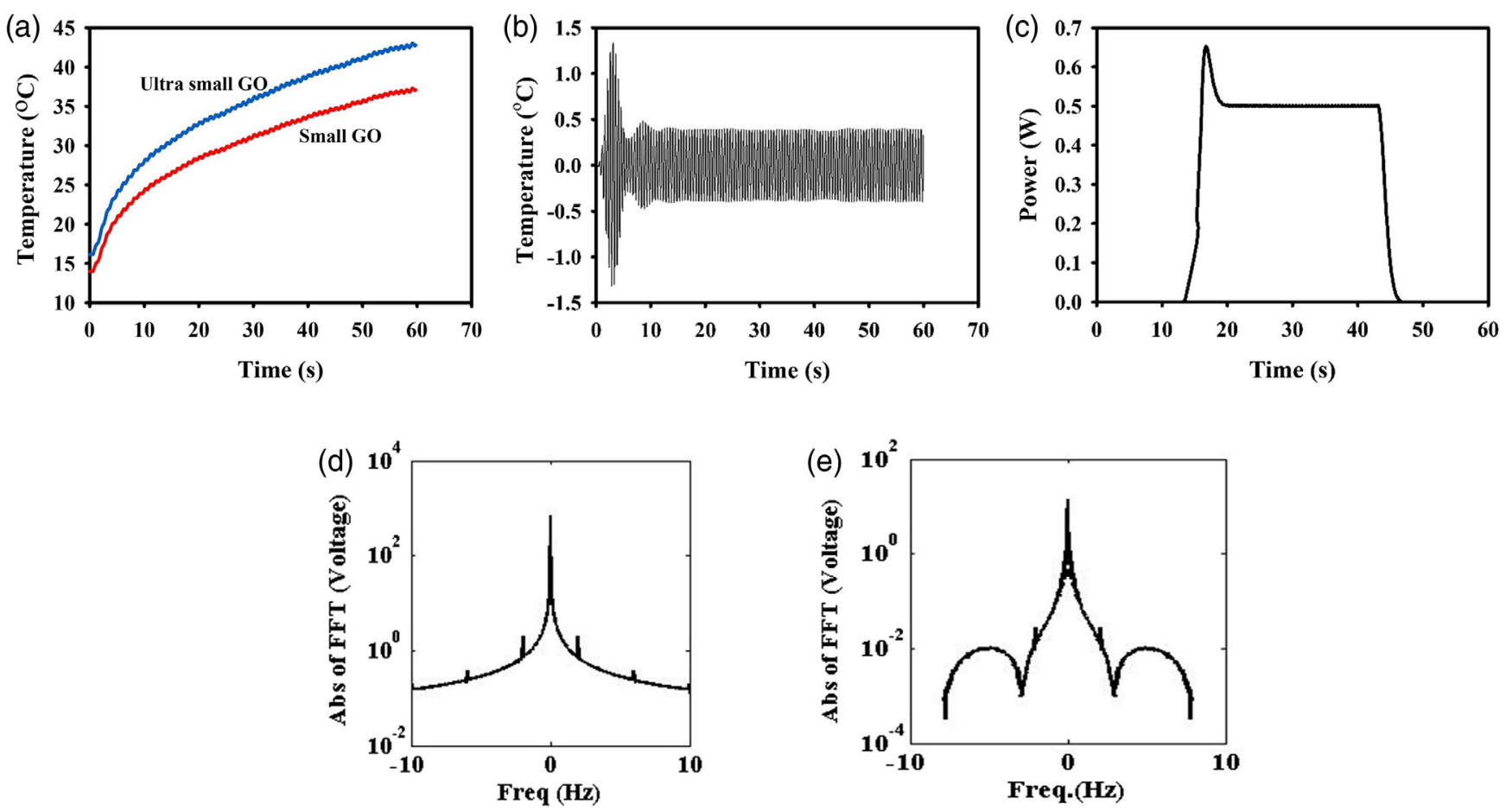

Fig. 3 (a) Temporal profile of temperature versus time of small and ultrasmall GO, (b) filtering result of plot A (ultrasmall GO), (c) temporal profile of power versus time measured by power meter, (d) FFT result of plot a small GO, and (e) FFT result of plot (c).

ratio of 0.12 and 0.21 were placed in a sample holder and irradiated by NIR laser pulse. An IR camera-monitored temperature rise for a minute [Fig. 3(a)], the profile was filtered with a band pass filter centered at $2 \mathrm{~Hz}$ [Fig. 3(b)] to ensure that the slope is not affected by intensity noise. To calculate $\varphi_{0}$, the time varying power was obtained over $50 \mathrm{~s}$ of pulse using a power meter [Fig. 3(c)]. Fast Fourier transform (FFT) of the temperature and power data confirms a 2-Hz signal frequency [Figs. 3(d) and $3(\mathrm{e})]$. As shown in Fig. 3, the laser power is $0.5 \mathrm{~W}$ so laser fluence was $1.77 \mathrm{~W} . \mathrm{cm}^{-2}$. By substituting $C_{w}=$ $4178.62 \mathrm{~J}(\mathrm{~kg} . \mathrm{K})^{-1}$ and $f=2 \mathrm{~Hz}$, the absorption coefficient $t$ and absorption cross section of GO shown in Table 3. Although numerous studies have been reported investigating the potential application of GO as a photoabsorber, the absorption cross section of these particles was unclear.

As presented in Table 3, the absorption cross section is dependent on the sheet size and chemical functional groups of GO. For instance, in the same sheet size, the absorption cross section and the absorption coefficient of GO nanoparticles decreased with increasing oxygen-to-carbon ratio. Moreover, by increasing the sheet size of GO with the same oxygen-to-carbon ratio, the absorption cross section increased for the same oxygen-to-carbon ratio. However, the absorption coefficient is slightly smaller for larger particles, probably owing to the higher scattering with the particle size and the concentration as discussed in theory, thereby reducing the temperature rise. With a decrease in the absorption coefficient for larger-sized particles, one would expect the absorption cross section to reduce. However, the factor of increase in molecular mass of the particle with an increase in the particle size is much greater than the factor of reduction in the absorption coefficient with an increase in the particle size, computed experimentally based on Eq. (11). Hence, there is an increase in the absorption cross-section values with increase in particle size. Absorption efficiency of the nanoparticles correlates directly with the absorption coefficient, with absorption efficiency reducing with the particle size due to the increase in scattering.

The effect of size distribution of GO sheets on the absorption cross section is another interesting parameter that should be considered. The size of each GO sample was determined by average mean radius $\langle a\rangle$, with a $\gamma$ as standard deviation in size uniformity that expressed as a percent of $\langle a\rangle$. To investigate the size distribution effect, the absorption cross section of five GO samples with same average mean radius $\langle a\rangle$ and different $\gamma$ was calculated as shown in Table 2. The effect of size distribution can be applied using the average over the appropriate size distribution function. The scattering cross section based on the Rayleigh model is proportional to $a^{6}$, so the average size value was found by replacing $\langle a\rangle^{6}$ by $\left\langle a^{6}\right\rangle$. By using the Gaussian distribution function $\left\langle a^{6}\right\rangle$ is given as

Table 2 Absorption cross section of GO: $C=0.25(\mathrm{mg} / \mathrm{ml}),(\mathrm{o} / \mathrm{c})=$ 0.21 in various size and oxygen to carbon ratio $(0.25 \mathrm{mg} / \mathrm{ml})$.

\begin{tabular}{|c|c|c|c|c|c|}
\hline $\begin{array}{l}\text { Sheet } \\
\text { size }(n m)\end{array}$ & $\begin{array}{c}\text { Standard } \\
\text { deviation } \\
\gamma(\%)\end{array}$ & $\rho_{g}\left(\frac{\mathrm{g}}{\mathrm{cm}^{3}}\right)$ & $m_{a}\left(\frac{\mathrm{kg}}{\mathrm{mol}}\right)$ & $\mu_{\alpha}\left(\frac{1}{\mathrm{~cm}}\right)$ & $\sigma\left(\mathrm{cm}^{2}\right)$ \\
\hline \multirow[t]{6}{*}{12} & 0 & 2.258 & 88.85 & 32206 & $1.90 \times 10^{-11}$ \\
\hline & 5 & 2.258 & 88.85 & 33493 & $1.98 \times 10^{-11}$ \\
\hline & 8 & 2.258 & 88.86 & 33172 & $1.96 \times 10^{-11}$ \\
\hline & 13 & 2.258 & 88.87 & 33816 & $1.99 \times 10^{-11}$ \\
\hline & 16 & 2.258 & 88.89 & 33172 & $1.96 \times 10^{-11}$ \\
\hline & 21 & 2.258 & 88.92 & 32850 & $1.94 \times 10^{-11}$ \\
\hline
\end{tabular}


Table 3 Absorption cross section of GO in various size and oxygen to carbon ratio $(0.25 \mathrm{mg} / \mathrm{ml})$.

\begin{tabular}{lccccc} 
Sheet size $(\mathrm{nm})$ & $\frac{o}{\mathrm{c}}$ & $\rho_{g}\left(\frac{\mathrm{g}}{\mathrm{cm}^{3}}\right)$ & $m_{a}\left(\frac{\mathrm{kg}}{\mathrm{mol}}\right)$ & $\mu_{\alpha}\left(\frac{1}{\mathrm{~cm}}\right)$ & $\sigma\left(\mathrm{cm}^{2}\right)$ \\
\hline 160 & 0.12 & 2.371 & 14252 & 33818 & $3.20 \times 10^{-9}$ \\
& 0.21 & 2.249 & 15728 & 24058 & $2.51 \times 10^{-9}$ \\
12 & 0.12 & 2.377 & 80.39 & 39555 & $2.11 \times 10^{-11}$ \\
& 0.21 & 2.258 & 88.85 & 32206 & $1.90 \times 10^{-11}$ \\
\hline
\end{tabular}

$\left\langle a^{6}\right\rangle=\frac{1}{\sqrt{2 \pi} \gamma} \int_{-\infty}^{\infty} a^{6} \exp \left[-\frac{(a-\langle a\rangle)^{2}}{2 \gamma^{2}}\right] \mathrm{d} a$.

This equation yields

$$
\left\langle a^{6}\right\rangle=\langle a\rangle^{6}+15\langle a\rangle^{4} \gamma^{2}+45\langle a\rangle^{2} \gamma^{4}+15 \gamma^{6} .
$$

Because $\gamma / a$ is small, the equation with good approximation can be rewritten as $15(\gamma /\langle a\rangle)^{2}$. The presented results in Table 3 show that the effect of the particle size distribution on the absorption cross-section is $<5 \%$. These results are in agreement with previous reports. ${ }^{40}$

As mentioned in the previous section, during computation of absorption cross section by this technique, the loss from heat diffusion process is minimized and the loss due to scattering is characterized. This increases the accuracy in the computation of absorption cross-section, as the uncertainty is quantified. Moreover, the result from this computation should be robust over different samples from the batch and across batches with different particle sizes. This is due to the results being obtained from the response of individual samples, with losses quantified. The deployment of Fourier-domain computation reduces the effect of external noise sources such as laser intensity noise on the results. The only issue with this technique is not taking the effect of diffraction into consideration during analysis. However, this drawback can be minimized by sonicating the sample batch for longer durations, thereby ensuring a large percentage of the particles used for experimentation have a radius smaller than $\lambda / 2 \pi$.

The presented results are in good agreement with other experimental studies reported by $\mathrm{Li}$ et al., ${ }^{19}$ Khan et al., ${ }^{18}$ and Hernandez et al. ${ }^{27}$ The absorptivity of graphene is higher than the calculated value for GO. This may have related to the isolation of $\mathrm{sp}^{2}$-bonded atoms in GO structure due to the presence of oxygen containing functional groups. ${ }^{19}$

\section{Conclusion}

In this study, absorption cross section of GO at $808 \mathrm{~nm}$ has been measured in the frequency domain by utilizing the Fourier analysis to reduce the laser intensity noise and other external variations, such that the chosen modulation frequency does not interfere with the environment. This measurement technique computes the absorption coefficient and absorptivity directly with minimization of various optical and thermal effects in the measurement process. However, in order to compute the absorption cross section, simulation and/or theoretical calculation is necessary to compute the number of particles in the sample. With proper care in experimentation, this technique may increase the robustness of computing absorption cross-section by reducing the variabilities of considered concentration and applied frequency. This is due to the fact that the losses are minimized and quantified with the absorption coefficient results computed on a per sample basis.

Moreover, this phototransducer characterizing technique can be extended to compute the absorption coefficient and absorption cross section of several different phototransducers. Several different single and multilayered phototransducers, which are being developed for effective and localized thermal treatments to kill cancerous cells. This technique can also be deployed to characterize these transducers per batch basis and account for variations in the batch, thereby optimizing the dosimetry of suspensions used for treatment.

From the experimental results, it is clear that the absorption cross section of GO nanoparticles reduces with increasing oxygen content in GO and reducing the particle size. However, the absorption coefficient of larger size particle is lesser owing to reduction in energy available for absorption with increase in scattering that accompanies with increase in particle size.

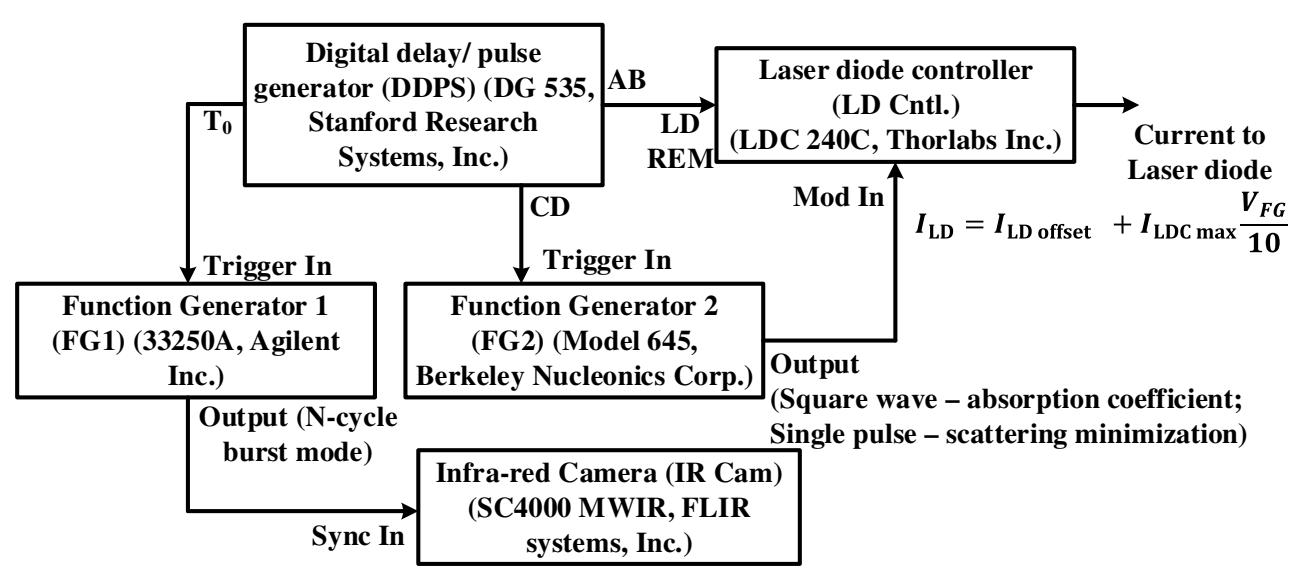

Fig. 4 Schematic of electronic controls that modulate the CW laser diode and IR camera of the photothermal therapeutic setup in Fig. 1, for computation of absorption coefficient in Fourier domain and estimate the scattering profile. 


\section{Appendix}

Schematic of electronic controls that modulate the $\mathrm{CW}$ laser diode and IR camera of the photothermal therapeutic setup in Fig. 1, for computation of absorption coefficient in Fourier domain and estimate the scattering profile (Fig. 4).

\section{Disclosures}

The authors would like to declare that this investigation was supported by Shahid Beheshti University G.C., research Grant No. 600/1426.

\section{References}

1. Z. Liu et al., "Graphene oxide based surface-enhanced Raman scattering probes for cancer cell imaging," Phys. Chem. Chem. Phys. 15(8), 29612966 (2013).

2. D. K. Chatterjee, P. Diagaradjane, and S. Krishnan, "Nanoparticlemediated hyperthermia in cancer therapy," Ther. Delivery 2(8), 1001-1014 (2011).

3. H. Huang-Chiao et al., "Spatiotemporal temperature distribution and cancer cell death in response to extracellular hyperthermia induced by gold nanorods," ACS Nano 4(5), 2892-2900 (2010).

4. J. Dory, "Apparatus for examining and localizing tumors using ultra sounds, comprising a device for localized hyperthermia treatment," Google Patents No. 4,658,828 (1987).

5. J. Chen et al., "Immuno gold nanocages with tailored optical properties for targeted photothermal destruction of cancer cells," Nano Lett. 7(5), 1318-1322 (2007).

6. C. Guo et al., "Photothermal ablation cancer therapy using homogeneous Cs x WO 3 nanorods with broad near-infra-red absorption," Nanoscale 5(14), 6469-6478 (2013).

7. L. R. Hirsch et al., "Nanoshell-mediated near-infrared thermal therapy of tumors under magnetic resonance guidance," Proc. Natl. Acad. Sci. U. S. A. 100(23), 13549-13554 (2003).

8. B. Jang et al., "Gold nanorod-photosensitizer complex for near-infrared fluorescence imaging and photodynamic/photothermal therapy in vivo," ACS Nano 5(2), 1086-1094 (2011).

9. C. S. Jin et al., "Ablation of hypoxic tumors with dose-equivalent photothermal, but not photodynamic, therapy using a nanostructured porphyrin assembly," ACS Nano 7(3), 2541-2550 (2013).

10. Y. Jin et al., "Graphene oxide modified PLA microcapsules containing gold nanoparticles for ultrasonic/CT bimodal imaging guided photothermal tumor therapy," Biomaterials 34(20), 4794-4802 (2013).

11. Z. Wang et al., "Fabrication and characterization of a triple functionalization of graphene oxide with $\mathrm{Fe}_{3} \mathrm{O}_{4}$, folic acid and doxorubicin as dual-targeted drug nanocarrier," Colloids Surf. B 106, 60-65 (2013).

12. J. Yang et al., "Smart drug-loaded polymer gold nanoshells for systemic and localized therapy of human epithelial cancer," Adv. Mater. 21(43), 4339-4342 (2009).

13. T. Ye et al., "Synthesis and optical properties of gold nanorods with controllable morphology," J. Phys.: Condens. Matter 28(43), 434002 (2016).

14. Y.-Y. Yu et al., "Gold nanorods: electrochemical synthesis and optical properties," J. Phys. Chem. B 101(34), 6661-6664 (1997).

15. Z. Zhang et al., "Mesoporous silica-coated gold nanorods as a lightmediated multifunctional theranostic platform for cancer treatment," Adv. Mater. 24(11), 1418-1423 (2012).

16. A. Abin-Fuentes et al., "Exploring the mechanism of biocatalyst inhibition in microbial desulfurization," Appl. Environ. Microbiol. 79(24), 7807-7817 (2013).

17. M. Hashemi et al., "Functionalized R9-reduced graphene oxide as an efficient nano-carrier for hydrophobic drug delivery," RSC Adv. 6(78), 74072-74084 (2016).

18. U. Khan et al., "Solvent-exfoliated graphene at extremely high concentration," Langmuir 27(15), 9077-9082 (2011).
19. D. Li and R. B. Kaner, "Graphene-based materials," Nat. Nanotechnol. 3, 101-105 (2008).

20. M. Yi et al., "Achieving concentrated graphene dispersions in water/ acetone mixtures by the strategy of tailoring Hansen solubility parameters," J. Phys. D: Appl. Phys. 46(2), 025301 (2012).

21. O. C. Compton and S. T. Nguyen, "Graphene oxide, highly reduced graphene oxide, and graphene: versatile building blocks for carbonbased materials," Small 6(6), 711-723 (2010).

22. S. Mikhailov, "Non-linear electromagnetic response of graphene," Europhys. Lett. 79(2), 27002 (2007).

23. H.-K. Jeong et al., "X-ray absorption spectroscopy of graphite oxide," Europhys. Lett. 82(6), 67004 (2008).

24. D. Mohammadrezaei et al., "In vitro effect of graphene structures as an osteoinductive factor in bone tissue engineering: a systematic review," J. Biomed. Mater. Res. Part A 106, 2284-2343 (2018).

25. M. Liu et al., "A graphene-based broadband optical modulator," Nature 474(7349), 64-67 (2011).

26. H. Huang et al., "Absorption and scattering cross section of regular black holes," J. Gravity 2014, 1-9 (2014).

27. Y. Hernandez et al., "High-yield production of graphene by liquid-phase exfoliation of graphite," Nat. Nanotechnol. 3, 563-568 (2008).

28. F. Liu and G. J. Smallwood, "Effect of aggregation on the absorption cross-section of fractal soot aggregates and its impact on LII modelling," J. Quant. Spectrosc. Radiat. Transfer 111(2), 302-308 (2010).

29. F. Liu et al., "Influence of polydisperse distributions of both primary particle and aggregate size on soot temperature in low-fluence LII," Appl. Phys. B: Lasers Opt. 83(3), 383-395 (2006).

30. P. K. Jain et al., "Calculated absorption and scattering properties of gold nanoparticles of different size, shape, and composition: applications in biological imaging and biomedicine," J. Phys. Chem. B 110(14), 72387248 (2006).

31. X. Zhou et al., "Chirality of graphene oxide-humic acid sandwich complex induced by a twisted, long-range-ordered nanostructure," J. Phys. Chem. C 120(45), 25789-25795 (2016).

32. A. K. Malde et al., "An automated force field topology builder (ATB) and repository: version 1.0," J. Chem. Theory Comput. 7(8), 4026-4037 (2011).

33. W. Huang, Z. Lin, and W. F. van Gunsteren, "Validation of the GROMOS 54A7 force field with respect to $\beta$-peptide folding," $J$. Chem. Theory Comput. 7(5), 1237-1243 (2011).

34. Y. Sefidbakht, O. Ranaei Siadat, and F. Taheri, "Homology modeling and molecular dynamics study on Schwanniomyces occidentalis alphaamylase," J. Biomol. Struct. Dyn. 35(3), 574-584 (2017).

35. B. Hess et al., "GROMACS 4: algorithms for highly efficient, load-balanced, and scalable molecular simulation," J. Chem. Theory Comput. 4(3), 435-447 (2008).

36. H. Hendrik Christoffel and H. C. van de Hulst, Light Scattering by Small Particles, Courier Corporation, New York (1957).

37. J. R. Frisvad, "Importance sampling the Rayleigh phase function," $J$. Opt. Soc. Am. A 28(8), 2436-2441 (2011).

38. J. T. Robinson et al., "Ultrasmall reduced graphene oxide with high near-infrared absorbance for photothermal therapy," J. Am. Chem. Soc. 133(17), 6825-6831 (2011).

39. M. Hashemi et al., "Normalization of doxorubicin release from graphene oxide: new approach for optimization of effective parameters on drug loading," Biotechnol. Appl. Biochem. 64, 443-442 (2016).

40. A. Cox, A. J. DeWeerd, and J. Linden, "An experiment to measure Mie and Rayleigh total scattering cross sections," Am. J. Phys. 70(6), 620625 (2002).

Mohadeseh Hashemi is a visiting scholar in the Division of Pharmaceutics and Biomedical Engineering at University of Texas at Austin, USA. She received her $\mathrm{PhD}$ in biomedical engineering from Tehran University, Iran at 2017 . Her research is focused on biomaterials and stimuli sensitive drug delivery system. She has more than 10 years of academic experience.

Biographies for the other authors are not available. 